\title{
Standards und Qualitätssicherung
}

\author{
Wissenschaftliche Erkenntnisse bedürfen der Umsetzung in die Praxis.
}

Die länger andauernde Nähe des Pflegefachdienstes zum Patienten führt zu einer ganz besonderen Position der Pflege im Alltag des Krankenhauses, wie in der ambulanten Versorgung. Sehr stark im Vordergrund steht der Beziehungsaspekt, aber zunehmend wird durch standardisierte Pflegeabläufe auch der Aspekt der Qualitätssicherung wichtig. Der Beitrag der Pflegewissenschaft wird hier ebenso deutlich, wie die Position von Pflegeexperten, die für die Umsetzung wissenschaftlicher Erkenntnisse in den Pflegealltag sorgen. Pflegerecht, Pflegemanagement und Pflegepraxis standen Ende Jänner im Mittelpunkt des Heilberufe Kongresses „Pflege 2009“ in Berlin.

Die demographische Entwicklung führt nicht nur zu einer Zunahme an speziellen Alterserkrankungen und besonders Demenzerkrankungen, sondern auch zum relativ neuen Phänomen des Demenzkranken im Akutkrankenhaus. So liegt die Prävalenz von Demenz heute beispielsweise in Deutschland bei einer Million. Sie wird sich verdoppeln, sagt Prof. Dr. Norbert Wrobel, Klinikum Bremen-Nord, Geriatrie und Frührehabilitation, voraus. Die Frage stelle sich, wie wir damit umgehen wollen. In der offiziellen Todesursachenstatistik findet sich Demenz nicht, als Hauptdiagnosen werden Herz-Kreislauf- und Tumorerkrankungen angeführt. Nach der Statistik spielt die Demenz also in der klinischen Versorgungsrealität keine Rolle. In der altersmedizinischen Versorgung, die primär auf die altersmedizinische Multimorbidität fokussiert, scheint die Diagnose Demenz jedoch sehr wohl auf. Dabei liegt der Anteil der Alzheimer-Demenz bei 60 Prozent, die Multiinfarkt-Demenz bei 30 Prozent und die sekundäre Demenz bei zehn Prozent. Kommt ein Patient mit einem Sturz einer Infektion oder ähnlichem ins Akutkrankenhaus wird jedoch nicht auf eine mögliche Demenz geachtet, stellte Wrobel fest. selbst dann nicht, wenn die Demenz die Ursache für die Akuterkrankung ist, wie beispielsweise der Sturz, der durch kognitive Probleme verursacht wurde, die Infektion, die durch unangemessene Kleidung bedingt wurde. Besonders problematisch seien nicht-kognitive Störungen, wie Wahn und Halluzinationen. „Demenzen“, so Wrobel, „müssen diagnostiziert werden. Auch wenn die Beratung aufwändig, aber wichtig ist, die Therapie bescheiden und die Entlassungsvorbereitung schwierig." Der medizinische Fortschritt sei zwar enorm - in der Diagnose und eventuell auch im Bereich einer Impfung zur Prävention. In den kommenden zehn Jahren sei eine völlig neue Situation zu erwarten. Derzeit ist die Demenz jedoch in der Akutklinik ein unterversorgtes Problem, was sich auch in der mangelnden Umsicht hinsichtlich der poststationären Versorgung manifestiere.

\section{Die pflegerische Sicht}

Wichtig in der Pflege Dementer sei der Beziehungsaufbau, nur so könne ein Zugang zum dementen Menschen gefunden werden, stellten Ina Henschke und Manuela Schroeder, Evangelisch-Freikirchliches Krankenhaus Rüdersdorf, fest. Der Demente braucht viel Orientierung und der Pflegende muss sich vor allem am Dementen orientieren - an seiner Langsamkeit, an seinen Bedürfnissen, an seiner Biographie. „Wir können davon ausgehen“, so

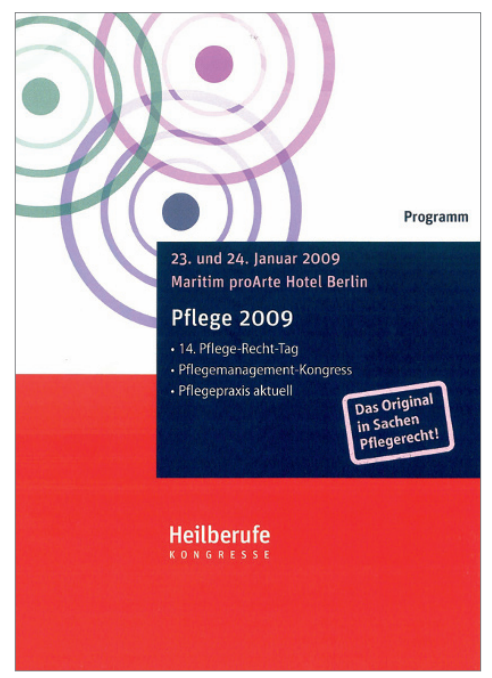

Henschke\&Schoeder, „dass der demenzkranke Mensch trotz seiner Beeinträchtigungen ein erlebnisfähiger, in seiner eigenen Welt lebender Mensch ist." Daher erlebt er auch die pflegerische Unterstützung immer als persönliche Erfahrung. Ebenso wichtig wie die empathische Pflege des Dementen sei aber auch die Betreuung seiner Angehörigen: Diese müssen gestärkt, entlastete und geleitet werden.

\section{Expertenstandards}

Mit Expertenstandards setzte das Deutsche Netzwerk für Qualitätsentwicklung in der Pflege (DNQP) ein Instrument zur Qualitätssicherung in Deutschland durch, das eine Vergleichbarkeit der Prozesse und Methoden ermöglicht. Bisher liegen Expertenstandards zu Dekubitusprophylaxe, Entlassungsmanagement, Schmerzmanagement, Sturzprophylaxe, Harnkontinenz und Pflege von Menschen mit chronischen Wunden vor. Der neueste Expertenstandard beschäftigt sich mit dem Ernährungsmanagement zur Sicherstellung und Förderung der oralen Ernährung in der Pflege. Er beschreibt, so Dr. rer. cur. Antje Tannen, Institut für Medizin-, Pflegepädagogik und Pflegewissenschaft der Charité Berlin, den pflegerischen Beitrag zum Ernährungsmanagement und zielt darauf ab, eine bedürfnis- und bedarfsgerechte Ernährung von kranken und pflegeabhängigen Menschen in allen Situationen der gesundheitlichen Versorgung zu sichern und zu fördern. Er beschreibt notwendige Strukturmerkmale der Einrichtungen und Kompetenzen ebenso wie wirksame Pflegeinterventionen und beobachtbare Pflegeergebnisse. Zum Ernährungsmanagement zählt ein Screening aller Bewohner/Patienten auf mögliche Anzeichen oder Risiken von Mangelernährung, ein tiefer gehendes Assessment auf Ursachen bei gefährdeten oder betroffenen Personen, die Planung und Steuerung multiprofessioneller Maßnahmen zur Ernährungsgestaltung, eine individuelle ernährungsbezogene Pflegeplanung, eine adäquate Interaktionsund Umgebungsgestaltung während der Mahlzeiten, eine individuelle Unterstützung des Bewohners/Patienten bei der Nahrungsaufnahme, eine adressatgerechte Beratung und eine fachgerechte Evaluation des Ernährungsmanagements mit Erfolgskontrolle. Mangelernährung geht mit einem erhöhten Mortalitäts- und Komplikationsrisiko einher, da sie zu einer Beeinträchtigung aller Stoffwechsel- und Organfunktionen führt. Daher ist eine Vermeidung von Mangelernährung ein wesentlicher Beitrag zur Gesunderhaltung. 